\title{
Ionic liquid dynamics in nanoporous carbon: A pore-size- and temperature-dependent neutron spectroscopy study on supercapacitor materials
}

\author{
Mark Busch $\odot,{ }^{1, *}$ Tommy Hofmann, ${ }^{2}$ Bernhard Frick $\odot,{ }^{3}$ Jan P. Embs, ${ }^{4}$ Boris Dyatkin $\odot,{ }^{5}$ and Patrick Huber $\odot 1, \uparrow$ \\ ${ }^{1}$ Institute of Materials Physics and Technology, Hamburg University of Technology, Eißendorfer Straße 42, 21073 Hamburg, Germany \\ ${ }^{2}$ Helmholtz-Zentrum Berlin für Materialien und Energie, Hahn-Meitner-Platz, 1, 14109 Berlin, Germany \\ ${ }^{3}$ Institut Laue-Langevin, 71 Avenue des Martyrs, 38000 Grenoble, France \\ ${ }^{4}$ Laboratory for Neutron Scattering and Imaging, Paul Scherrer Institute, 5232 Villigen, Switzerland \\ ${ }^{5}$ Department of Materials Science and Engineering, A. J. Drexel Nanomaterials Institute, Drexel University,
} Philadelphia, Pennsylvania 19104, USA

(Received 21 October 2019; revised manuscript received 8 April 2020; accepted 6 May 2020; published 27 May 2020)

\begin{abstract}
The influence of spatial confinement on the thermally excited stochastic cation dynamics of the roomtemperature ionic liquid 1-N-butylpyridinium bis-[(trifluoromethyl)sulfonyl]imide ([BuPy] $\left.\left[\mathrm{Tf}_{2} \mathrm{~N}\right]\right)$ inside porous carbide-derived carbons with various pore sizes in the sub- to a few nanometer range is investigated by quasielastic neutron spectroscopy. Using the potential of fixed window scans, i.e., scanning a sample parameter, while observing solely one specific energy transfer value, an overview of the dynamic landscape within a wide temperature range is obtained. It is shown that already these data provide a quite comprehensive understanding of the confinement-induced alteration of the molecular mobility in comparison to the bulk. A complementary, more detailed analysis of full energy transfer spectra at selected temperatures reveals two translational diffusive processes on different time scales. Both are considerably slower than in the bulk liquid and show a decrease of the respective self-diffusion coefficients with decreasing nanopore size. Different thermal activation energies for molecular self-diffusion in nanoporous carbons with similar pore size indicate the importance of pore morphology on the molecular mobility, beyond the pure degree of confinement. In spite of the dynamic slowing down we can show that the temperature range of the liquid state upon nanoconfinement is remarkably extended to much lower temperatures, which is beneficial for potential technical applications of such systems.
\end{abstract}

DOI: 10.1103/PhysRevMaterials.4.055401

\section{INTRODUCTION}

Room-temperature ionic liquid electrolytes in combination with porous carbon electrodes are promising candidates for electric double layer capacitors (EDLCs), which are commonly referred to as super- or ultracapacitors [1-5]. These capacitors benefit from high energy densities on a par with those of batteries along with the superior power densities of conventional capacitors and thus offer efficient electric energy storage and conversion systems for a variety of applications. The use of ionic liquids has numerous advantages as compared to conventional aqueous or solvated organic salt electrolytes. Most importantly, they possess a wide electrochemical window, good temperature stability, and low volatility $[1,3,6-$ 8]. Nanoporous carbons distinguish themselves by a welltunable pore size, shape, and surface chemistry. Furthermore, they possess a high electrical conductivity, good (electro-) chemical stability, and a large specific surface area, making them well-suited electrode materials [9]. Although these systems are a very active field of research, the actual technical implementation as high-performance supercapacitors remains

\footnotetext{
*mark.busch@tuhh.de

†patrick.huber@tuhh.de
}

challenging. One of the reasons is the relatively high viscosity of ionic liquids $[8,10,11]$, resulting in slow ion dynamics $[8,12]$. While some studies find increased dynamics in the case of carbon nanoconfinement [13-16], others see a clear slowdown [17-20]. Additionally, ionic liquids tend to layer at solid interfaces [21,22]. As a consequence, they often form immobile layers at pore walls and molecules within micropores thus frequently do not exhibit any appreciable diffusive motion $[13,14,20,23,24]$, while even confinement-induced freezing has been reported [25]. This further reduces the amount of mobile ions in the nanopores and thus potentially decreases the supercapacitor performance, since (dis-)charging involves diffusive processes [26].

To resolve these uncertainties, more profound knowledge about the self-diffusion properties of ionic liquids in carbon nanoconfinement must be obtained. In this context, the influence of different nanopore sizes on the dynamics is not only of importance with respect to possible immobilized ionic surface layers but also because of the dependence of the capacitance on the pore diameter. It has been found that the capacitance of ionic liquid based electrical double layer capacitors depends not monotonically, but rather in an oscillatory manner, on the pore size [27-29]. Thus, additional research efforts must find the optimal nanopore width that yields the best compromise between capacitance and molecular mobility of the ionic 
liquid molecules. The latter, however, usually decreases with lower temperatures and the temperature range of the liquid phase is often quite limited for bulk ionic liquids, especially towards low temperatures $[3,8,10,13,30]$. But nanoconfinement of ionic liquids is known to change the phase transition behavior [31,32].

To address these fundamental issues, we present a quasielastic neutron scattering investigation on the selfdiffusion dynamics of a room-temperature ionic liquid confined in the nanopores of carbide-derived carbons (CDCs) as a function of pore size over a wide temperature range. In a first step, we analyze the data of so-called fixed window scans, which allow us to rapidly scan a broad temperature range and provide a first but already detailed overview of the dynamics in our systems. In a second step, these findings are complemented at selected temperatures by the full spectroscopic information from two spectrometers with complementary energy resolution and dynamic range, providing further insights into certain dynamic peculiarities of the nanoconfined ionic liquid.

\section{SAMPLE PREPARATION \& CHARACTERIZATION}

Porous CDC microparticles were obtained using previously reported procedures [33]. Silicon carbide ( $\mathrm{SiC})$, molybdenum carbide $\left(\mathrm{Mo}_{2} \mathrm{C}\right)$, and boron carbide $\left(\mathrm{B}_{4} \mathrm{C}\right)$ microparticles $(1-5 \mu \mathrm{m}$ wide) were used as precursors. These were etched with $\mathrm{Cl}_{2}$ gas at specific temperatures to remove the metal atoms and structure sample-specific nanoporosity and were subsequently annealed in $\mathrm{H}_{2}$ gas at $600{ }^{\circ} \mathrm{C}$. $\mathrm{SiC}$ was etched at $1000{ }^{\circ} \mathrm{C}$ with $\mathrm{Cl}_{2}$ and following the $\mathrm{H}_{2}$ annealing, it was oxidized in air at $425^{\circ} \mathrm{C}$ and annealed under high vacuum ( $10^{-6}$ torr) at $1400{ }^{\circ} \mathrm{C}$ (labeled as ' $\mathrm{SiC}-2$ '). $\mathrm{Mo}_{2} \mathrm{C}$ was etched at $900{ }^{\circ} \mathrm{C}$ with $\mathrm{Cl}_{2}$ and after the $\mathrm{H}_{2}$ annealing it underwent identical oxidation and annealing conditions as in the case of $\mathrm{SiC}$ (labeled as ' $\mathrm{MoC}-15$ '). After the etching of $\mathrm{B}_{4} \mathrm{C}$ with $\mathrm{Cl}_{2}$ at $900{ }^{\circ} \mathrm{C}$ and the following $\mathrm{H}_{2}$ annealing, it underwent either the same oxidation and annealing conditions as above (labeled as 'BC-6') or vacuum annealing only, without preceding air oxidation step (labeled as 'BC-6-no'). While the vacuum annealing final step removes all surface functional groups and makes electrode surface chemistries of each system near identical $[34,35]$, the preceding steps yielded different pore widths.

Figure 1 shows the pore size distribution of the CDCs, as obtained from the nitrogen sorption isotherms, employing quenched solid density functional theory models [36]. The numbers at the end of the sample identifiers indicate the largest nanometer-sized pore diameter determined for the respective specimen.

Subsequently the nanopores of the carbide-derived carbons are filled with the room-temperature ionic liquid 1-Nbutylpyridinium bis-[(trifluoromethyl)sulfonyl]imide, in the following shortly denoted as $[\mathrm{BuPy}]\left[\mathrm{Tf}_{2} \mathrm{~N}\right]$ and whose bulk molecular dynamics have been already extensively studied [37-41]. The structure formulas of the cation and anion are depicted in Fig. 2. Filling of the nanoporous carbons with the ionic liquid for the neutron spectroscopy experiments is a crucial process. Since the aim is to study the dynamics of the ionic liquid confined in the nanopores, any bulk liquid

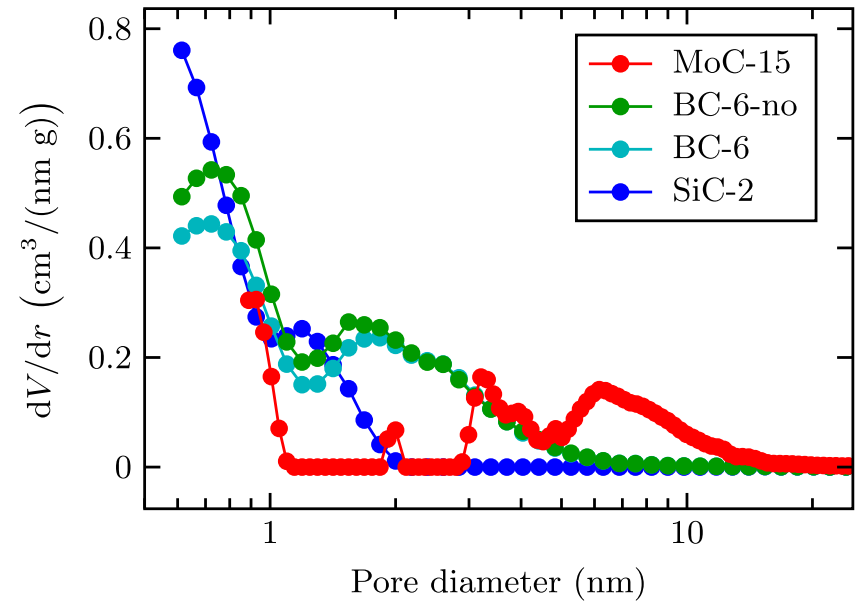

FIG. 1. Pore size distribution of the carbide-derived nanoporous carbon samples, as obtained using a density functional theory based analysis of the nitrogen sorption isotherms (lines between the points are guides for the eye.).

between the carbon grains must be avoided, as that could overwhelm the measurement signal from the confined liquid. Therefore, for each sample, only an amount of ionic liquid equaling the pore volume obtained from the nitrogen gas sorption data analysis is used. That volume of $[\mathrm{BuPy}]\left[\mathrm{Tf}_{2} \mathrm{~N}\right]$ is mixed with ethanol ( $\geqslant 99.9 \%$ purity) in a volume ratio of 1:2 and afterwards blended with the respective CDC. The compound is stored in a drying cabinet for 2.5 hours at $75{ }^{\circ} \mathrm{C}$ and $8 \mathrm{mbar}$, to remove the ethanol and accelerate the capillary imbibition of the highly viscous ionic liquid into the nanopores. Pore filling of the now visually dry powder is verified gravimetrically.

\section{METHODS}

Quasielastic neutron scattering (QENS) experiments have been performed at the time-of-flight spectrometer FOCUS at the Paul Scherrer Institute (Villigen, Switzerland) and the neutron backscattering spectrometer IN16B at the Institut LaueLangevin (Grenoble, France) [42]. At FOCUS neutrons with a wavelength of $6.00 \AA$ were used, giving access to an energy transfer range of $\pm 1.0 \mathrm{meV}$ with a simultaneously accessible wave vector transfer $Q$ between $0.32 \AA^{-1}$ and $1.64 \AA^{-1}$ and an energy resolution of around $39 \mu \mathrm{eV}$ (FWHM). IN16B was used in its low-background position, with an incident neutron wavelength of $6.27 \AA$. Here, the maximum energy transfer $\Delta E$ is $\pm 31 \mu \mathrm{eV}$, with a resolution of around $0.85 \mu \mathrm{eV}$ (FWHM) and a $Q$ range from $0.19 \AA^{-1}$ to $1.90 \AA^{-1}$.
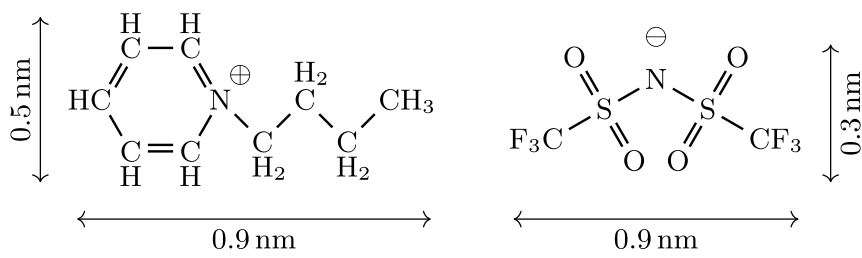

FIG. 2. Structure formula of the cation (left) and the anion (right) of the ionic liquid $[\mathrm{BuPy}]\left[\mathrm{Tf}_{2} \mathrm{~N}\right]$ with estimate of the ions' size. 

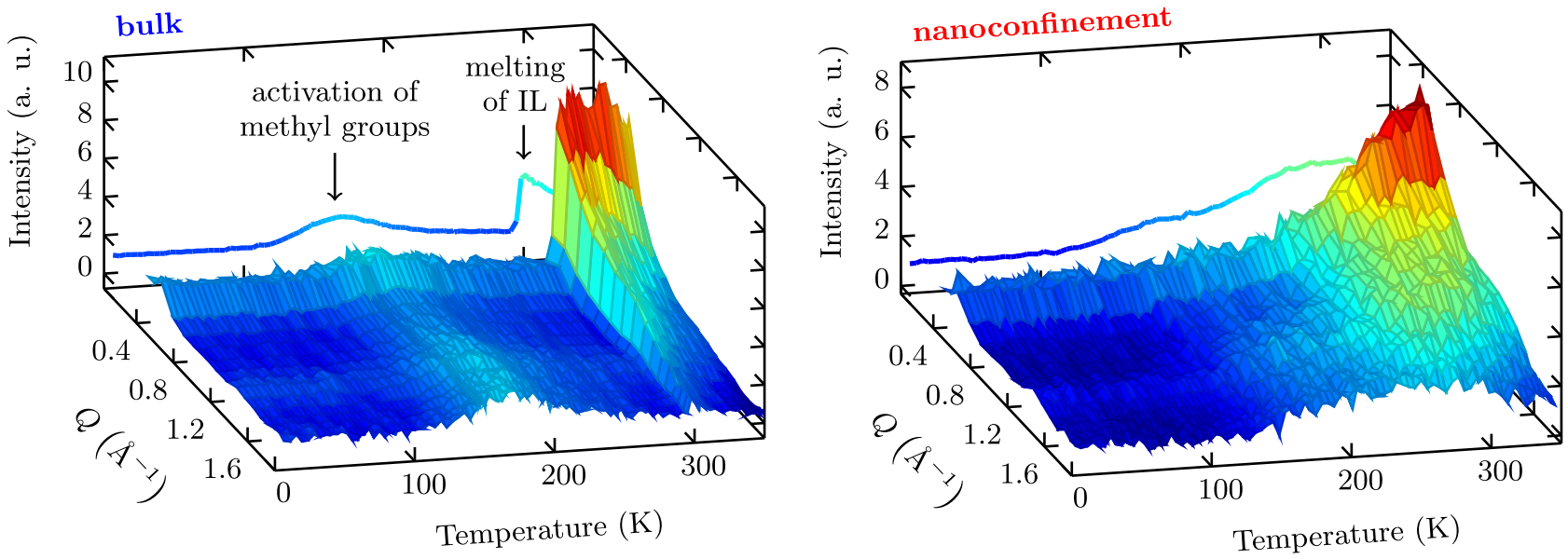

FIG. 3. Intensity of neutrons with an energy transfer of $\pm 2 \mu \mathrm{eV}$, when scattered at (left) bulk [BuPy][Tf $\left.{ }_{2} \mathrm{~N}\right]$ and (right) in the nanoconfinement of MoC-15 as a function of wave vector transfer $Q$, while scanning the temperature from $2 \mathrm{~K}$ to $355 \mathrm{~K}$ with a rate of $1 \mathrm{~K} / \mathrm{min}$. Back panel: Intensity averaged over the available $Q$ range.

At IN16B not only full quasielastic spectra at selected temperatures were acquired but also so-called fixed windows scans were performed. The latter enables one to quickly scan a very broad temperature range, while observing the intensity of a fixed $\Delta E$ channel of the spectrum [43]. In case of the full quasielastic spectra data from an empty cell measurement was subtracted from the specimen data.

The incoherent neutron scattering cross section of the cation of the ionic liquid is 1124.24 barn, while the one of the anion is with only 0.53 barn considerably smaller [44]. As a result it is mainly the dynamics of the cation that is probed by the inelastic neutron scattering methods employed in this work. As for the type of cation dynamics we can expect contributions from local motions (ring wagging, segmental rotation in the butyl side chain, methyl group rotation) and from center-of-mass diffusion. In general local motions are characterized in QENS by a $Q$ independence of the line broadening, whereas diffusive processes show a pronounced $Q$ dependence.

At both spectrometers the sample holder was placed in a cryostat enabling a temperature control of the specimens. All samples were encased in flat, slab-shaped aluminium sample holders. The primary data reduction, detector efficiency calibration utilizing a vanadium standard, and corrections like those concerning self-shielding were done using the DAVE [45] (FOCUS) and the LAMP [46] (IN16B) software packages. In principal after these corrections the data can be converted into the dynamic structure factor $S(Q, \omega)$ which can then be compared to theoretical models after convolution with the instrumental resolution.

\section{EXPERIMENTAL RESULTS \& DISCUSSION}

\section{A. Fixed window scans}

To get an overview of the various dynamic processes setting in at different temperatures in the systems under investigation, elastic (EFWS) and inelastic fixed window scans (IFWS) are conducted in a temperature range from $2 \mathrm{~K}$ to $355 \mathrm{~K}$. While heating the specimens with a rate of $1 \mathrm{~K} / \mathrm{min}$, alternately the intensity of the elastically scattered neutrons, i.e., $\Delta E=0 \mu \mathrm{eV}$ and those having experienced an energy change of $\Delta E= \pm 2 \mu \mathrm{eV}$ is measured. Figure 3 shows the acquired data from such an IFWS at the example of the bulk $[\mathrm{BuPy}]\left[\mathrm{Tf}_{2} \mathrm{~N}\right]$ (left panel), which is measured as a reference for the subsequent analysis of the respective cation dynamics in nanoconfinement (right panel). This comparison already impressively illustrates the confinement-induced alterations in the phase transition behavior, as well as the $Q$ dependence of the data at different temperatures, giving first hints on the nature of the molecular dynamics. A further qualitative overview of the temperature-dependent activation of dynamic processes is given by the averaged intensity over the $Q$ range between $0.44 \AA^{-1}$ and $1.90 \AA^{-1}$, that is depicted in the back panel of Fig. 3, as well as in Fig. 4 for the bulk [BuPy] $\left[\mathrm{Tf}_{2} \mathrm{~N}\right]$ and confined inside the nanopores of the CDCs with different pore sizes. Here, all intensities, i.e., the ones of the elastic, as well as those of the inelastic fixed window scans, are normalized to the elastic intensity at $2 \mathrm{~K}$.

For the bulk ionic liquid, around $100 \mathrm{~K}$ a drop in the elastic intensity [see Fig. 4(a)] occurs, while that in the $\pm 2 \mu \mathrm{eV}$ channels [see Fig. 4(b)] starts to rise considerably. Since bulk $[\mathrm{BuPy}]\left[\mathrm{Tf}_{2} \mathrm{~N}\right]$ is at this temperature still in its crystalline state, this is not the result of any diffusive motion of the molecules as a whole but originates in the activation of the dynamics of subsets of atoms, like those of methyl groups [39] and segmental rotations of the butyl group. When with increasing temperature the dynamics accelerate, the energy transfer between the neutrons and the sample is distributed over a growing energy range and consequently the intensity at the observed $\Delta E= \pm 2 \mu \mathrm{eV}$ energy transfer first increases and then decreases. At approx. $295 \mathrm{~K}$ the sudden drop/rise in the elastic/inelastic intensity indicates the melting of the bulk ionic liquid $[47,48]$. The situation, however, is completely different in the case of the nanoconfined [BuPy] $\left[\mathrm{Tf}_{2} \mathrm{~N}\right]$. The rise of the inelastic intensity at $\Delta E= \pm 2 \mu \mathrm{eV}$ from $100 \mathrm{~K}$ on is less steep than in the bulk. But more interestingly, there is no abrupt intensity increase, as for the melting of the bulk liquid, but the intensity starts to gradually rise already at 


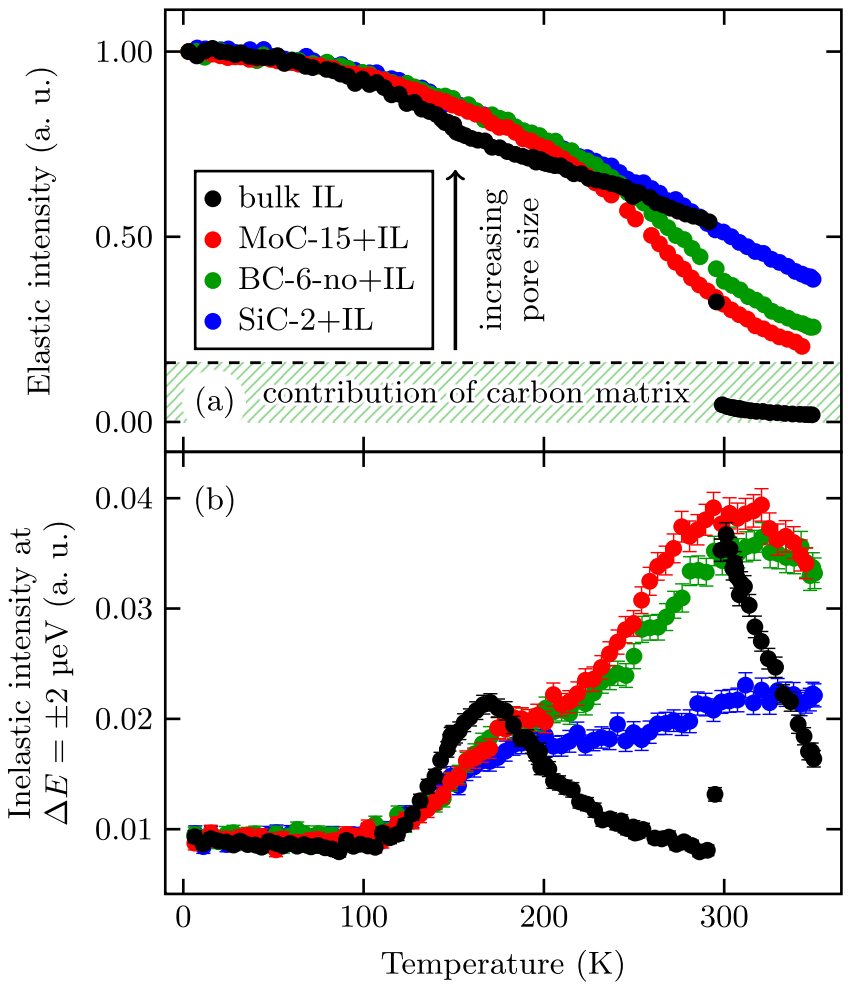

FIG. 4. Fixed window scans in a temperature range from $2 \mathrm{~K}$ to $355 \mathrm{~K}$ with a heating rate of $1 \mathrm{~K} / \mathrm{min}$ for the ionic liquid in bulk and in nanoconfinement of different pore sizes (intensity averaged over a $Q$ range from $0.44 \AA^{-1}$ to $1.90 \AA^{-1}$ ): (a) Elastic fixed window scans, i.e., $\Delta E=0 \mu \mathrm{eV}$; (b) inelastic fixed window scans at an energy transfer of $\Delta E= \pm 2 \mu \mathrm{eV}$.

much lower temperatures, such that there are two overlapping peaks. Furthermore, there is a clear pore size dependence. The maximum of the two peaks is successively shifted towards higher temperatures with decreasing nanopore size, while the maximum intensity of the peaks is decreasing. The latter, together with the inverse trend in the elastic intensity, suggests a growing part of immobile ions with respect to their centerof-mass diffusion.

The finding of a gradual melting in the confined versus a discontinuous melting in the bulk state could be related to interfacial melting, i.e., the gradual mobilization of the molecules starting from the carbon pore wall upon heating. Analogous observations have been made for many other liquids in confined geometries [49-51], in particular also at graphitic interfaces [52]. Furthermore, the pore-size dependence of the melting point reduction is a common phenomenon for liquids in porous materials [51,53-55]. The Gibbs-Thomson equation, which is strictly valid only for large pores, predicts that the melting point shift scales with the inverse of the pore diameter, where the shift direction depends on the type of interaction of the confined material with the pore wall surface $[55,56]$. Due to the broad pore-size distribution of the CDCs (see Fig. 1), it can be expected that this effect significantly contributes to the observed melting transition broadening.

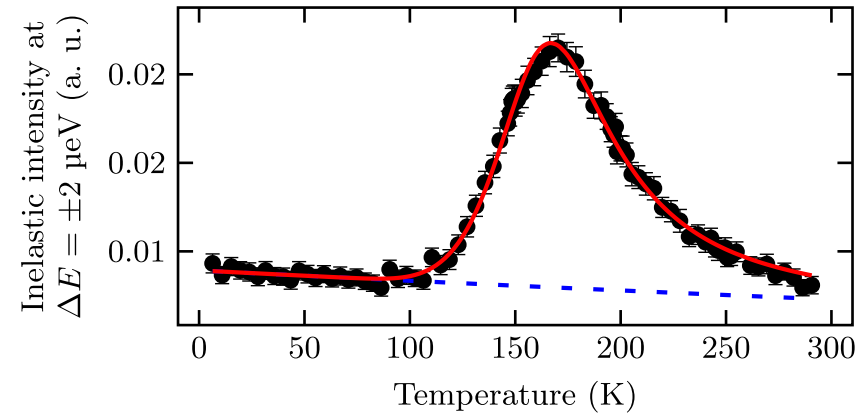

FIG. 5. Fit to the elastic fixed window scan data of the lowtemperature dynamics of the bulk ionic liquid with the model as delineated in the text.

\section{Low-temperature cation dynamics of the bulk ionic liquid}

To further interpret the above findings, first the lowtemperature dynamics of the bulk ionic liquid are analyzed. For localized motions the incoherent dynamic structure factor can in the simplest case be written as

$$
\begin{aligned}
& S^{\text {local }}(Q, T, \omega) \\
& \quad=A(Q) \delta(\omega)+(1-A(Q)) \cdot \frac{1}{\pi} \frac{\gamma_{1}(Q, T)}{\gamma_{1}^{2}(Q, T)+\omega^{2}},
\end{aligned}
$$

where the elastic incoherent structure factor (EISF) $A(Q)$ gives information about the geometry of these motions [57]. $A(Q)$ is considered here to be temperature independent. Equation (1) is convoluted with the instrumental resolution and therefore also dynamic processes slower than the resolution of the respective instrument may appear to be elastic. The spectral function is assumed in Eq. (1) to be a Lorentzian with the half width at half maximum (HWHM) $\gamma_{1}$ that is given by the inverse of the relaxation time $\tilde{\tau}^{\text {local }}$ of the corresponding dynamics. This relaxation time is presumed to have an Arrhenius temperature dependence $[43,58]$, i.e.,

$$
\tilde{\tau}^{\text {local }}(T)=\tilde{\tau}_{\infty}^{\text {local }} \cdot \exp \left(\frac{E_{\mathrm{a}}^{\text {local }}}{R T}\right),
$$

where $R$ is the universal gas constant, $\tilde{\tau}_{\infty}^{\text {local }}$ the high temperature limit of the relaxation time, and $E_{\mathrm{a}}^{\text {local }}$ the activation energy of this dynamical process. For the methyl group dynamics a systematic $Q$ dependence of the HWHM is neither expected [59] nor found here (see left panel of Fig. 3). Therefore, the intensity, integrated over all for the fixed window scans available $Q \mathrm{~s}$, is analyzed. Since furthermore, the expression in Eq. (1) is evaluated for $\omega= \pm 2 \mu \mathrm{eV} / \hbar=: \omega_{\text {IFWs }}$ only, the temperature-dependent intensity of the fixed window scan, as depicted in Figs. 4 and 5, is described by

$$
I(T)=e^{-a \cdot T} \cdot\left[I_{0} \cdot \frac{1}{\pi} \frac{\tilde{\tau}^{\text {local }}(T)}{1+\left(\tilde{\tau}^{\text {local }}(T)\right)^{2} \cdot \omega_{\mathrm{IFWS}}^{2}}+c_{0}\right] .
$$

$I_{0}$ is an intensity factor and $c_{0}$ a constant offset. Both are containing a contribution originating in the convolution of the dynamic structure factor [Eq. (1)] with the resolution function of the instrument [43]. The exponential prefactor describes the temperature dependence of the Debye-Waller factor [60], determined by a respective fit to the data in the low-temperature region of the corresponding EFWS [cp. 
Fig. 4(a)], before any molecular dynamics aside from lattice vibrations sets in. The constant offset $c_{0}$ is ascertained in the same temperature region but from the IFWS. As can be seen from Fig. 5, the employed model fits the measured data very well. It yields an activation energy of $9.7 \mathrm{~kJ} / \mathrm{mol}$ for these localized motions. This value is larger than those obtained by Burankova et al. [39], employing a three-site jump and a rotational diffusion model, yielding $6.5 \mathrm{~kJ} / \mathrm{mol}$ and $7.0 \mathrm{~kJ} / \mathrm{mol}$, respectively, for the analysis of an EFWS at the bulk $[\mathrm{BuPy}]\left[\mathrm{Tf}_{2} \mathrm{~N}\right]$ with a deuterated pyridinum ring. This is however not surprising, because our cations are fully protonated and thus the determined activation energy is to be considered as an average over all localized dynamics of the molecules. Beneath methyl group dynamics and segmental rotations of the butyl chain, this includes motions of the pyridinium ring.

\section{Nanoconfined cation dynamics}

In a next step, the above approach is extended to analyze the molecular dynamics of the ionic liquid confined in the nanoporous carbon over the whole measured temperature range. Contrary to the bulk liquid, the localized dynamics and the ones ascribed to the translational diffusion of the molecule, cannot be analyzed separately, since their measurement signals within the IFWS overlap with respect to their temperature range. As a consequence the following ansatz combining both dynamics is chosen to describe the intensity of the IFWS:

$$
I(Q, T, \Delta E)=e^{-a \cdot T} \cdot\left[I_{0}(Q) \cdot S^{\mathrm{dyn}}(Q, T, \Delta E)+c_{0}(Q)\right],
$$

where

$$
S^{\mathrm{dyn}}(Q, T, \Delta E)=S^{\text {local }}(T, \Delta E) \otimes S^{\mathrm{global}}(Q, T, \Delta E) .
$$

The dynamic structure factor for the center-of-mass diffusion of the cation,

$$
S^{\text {global }}(Q, T, \Delta E)=\frac{1}{\pi} \frac{\Gamma_{2}(Q, T)}{\Gamma_{2}^{2}(Q, T)+\Delta E^{2}},
$$

depends on the wave vector transfer $Q$, while the component considering the localized dynamics, $S^{\text {local }}(T, \Delta E)$, is fully analogous to the one for the bulk ionic liquid [see Eqs. (1) and (2)] with a $Q$-independent half width of the spectral function. Because however, the number of free parameters would exceed the number of equations to be solved, further assumptions have to be taken into account: Embs et al. [37] found in a QENS study of the bulk [BuPy] $\left[\mathrm{Tf}_{2} \mathrm{~N}\right]$ that the HWHM $\Gamma_{2}$ of the global component obeys the SingwiSjölander jump-diffusion model [61], i.e.,

$$
\Gamma_{2}(Q)=\frac{\hbar D Q^{2}}{1+D Q^{2} \tau_{0}} .
$$

In addition, the self-diffusion coefficient $D$ follows an Arrhenius-like temperature dependence [37],

$$
D(T)=D_{\infty} \cdot \exp \left(-\frac{E_{\mathrm{a}}^{\mathrm{global}}}{R T}\right),
$$

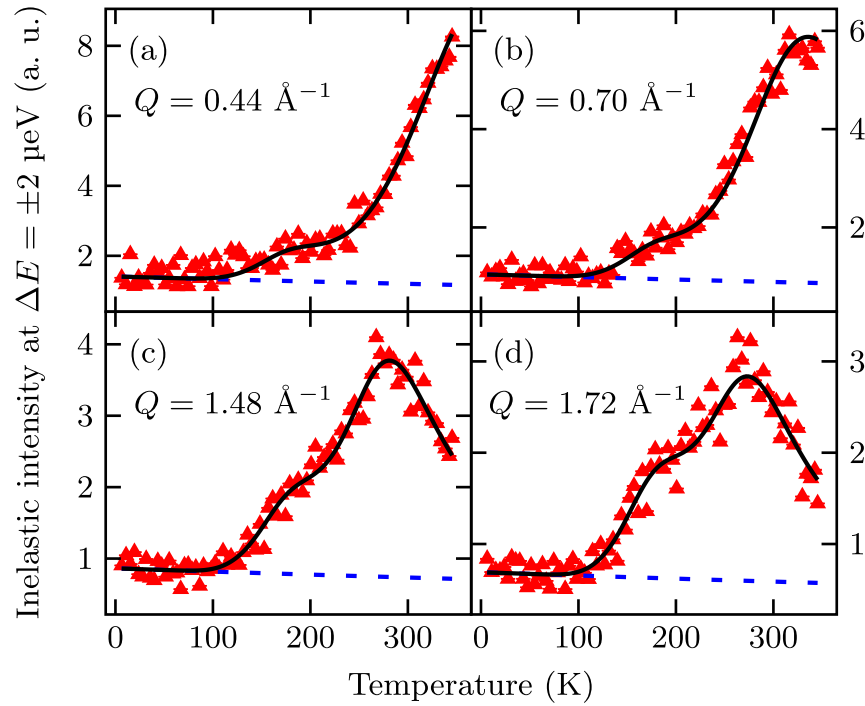

FIG. 6. Inelastic fixed window scan at sample MoC-15+IL at four exemplary wave vector transfers $Q$ with the corresponding results of the fit to the data, which is performed for all $16 Q$ simultaneously with a common parameter set and with the model as described in the text.

and their data suggest that this also holds for the residence time $\tau_{0}$, with

$$
\tau_{0}(T)=\tau_{\infty} \cdot \exp \left(\frac{E_{\mathrm{a}}^{\text {global }}}{R T}\right) .
$$

This model is fitted to the $16 Q$ values of the IFWS data simultaneously with a common set of parameters: the hightemperature limits of the self-diffusion coefficient and residence time, $D_{\infty}$ and $\tau_{\infty}$, of the relaxation time of the localized motions, and the activation energies of the localized and diffusive dynamics. The intensity factor $I_{0}$ and the EISF $A$ are individual for each $Q$. The exponential prefactor and the background are ascertained as described above for the bulk liquid, but the constant offset $c_{0}$ is determined for each curve, separately.

This model reproduces the temperature dependence of the IFWS intensity very well, as can be exemplary seen in Fig. 6, showing the fit to the measurement for sample MoC-15+IL at selected wave vector transfers $Q$. Table I lists the activation energies for the translational diffusion of the whole cation,

TABLE I. Activation energies of the localized dynamics and center-of-mass diffusion of [BuPy] in bulk, as well as in the nanoconfinement of the CDC samples (ordered by decreasing pore size). All data are obtained from the inelastic fixed window scans, except the bulk value of $E_{\mathrm{a}}^{\text {global }}$ (taken from Refs. [37,38,40,41]).

\begin{tabular}{lcc}
\hline \hline & $\begin{array}{c}\text { Localized dynamics } \\
E_{\mathrm{a}}^{\text {local }}(\mathrm{kJ} / \mathrm{mol})\end{array}$ & $\begin{array}{c}\text { Diffusive motion } \\
E_{\mathrm{a}}^{\text {global }}(\mathrm{kJ} / \mathrm{mol})\end{array}$ \\
\hline bulk IL & 9.7 & $12.3-14.8$ \\
MoC-15+IL & 7.1 & 15.1 \\
BC-6-no+IL & 6.9 & 14.9 \\
SiC-2+IL & 6.0 & 12.0 \\
\hline \hline
\end{tabular}


as well as of its localized dynamical processes, obtained from the above analysis. The activation energy of the local motions turns out to be notably smaller in nanoconfinement compared to bulk and it furthermore diminishes with decreasing pore size. For the activation energy of the center-of-mass diffusion of the cation the same trend is observed: Although the magnitude of the activation energy is for all pores within the range determined for the bulk [37], it decreases systematically with the pore size. The found diminution of the activation energy in the nanopore confinement may appear counterintuitive on the first view, because the maxima of the curves from the IFWS (see Fig. 4) are successively shifted to higher temperatures with decreasing pore size. But since the relaxation time $\tilde{\tau}_{\infty}^{\text {local }}$ [see Eq. (2)] in contrast increases at the same time, the resulting line width $\gamma_{1}$ decreases nevertheless. Such a confinement-induced reduction of the methyl group relaxation velocity is also known from substances, like toluene [62,63] and polymers [63,64], where it is ascribed to the interaction of an immobilized surface layer with the confining pore walls. This assumption is supported by the increasing relaxation time with decreasing pore size, entailing an augmentation of the surface-to-volume ratio and thus strengthening the role of surface-interaction-induced effects. Similar to the relaxation time, also the residence time $\tau_{0}$ concerning the diffusion of the cation as a whole increases, while the respective selfdiffusion coefficient $D$ decreases, with decreasing nanopore size (see open circles in Fig. 7). Thus the corresponding line width $\Gamma_{2}$ [see Eq. (7)] is smaller for narrower pores and consequently the maximum of the fixed window scan intensity in Fig. 4 is shifted to higher temperatures. Such a behavior is intelligible, because the increasing confinement in smaller pores hinders the molecular motions of the cation and thus prolongates the corresponding relaxation times, while slowing down the diffusion. This effect is further intensified by a growing immobile portion of molecules at the pore walls, decreasing the volume of mobile ions, as will be discussed below.

\section{B. Full quasielastic spectra}

For a more detailed analysis of the cation dynamics of $[\mathrm{BuPy}]\left[\mathrm{Tf}_{2} \mathrm{~N}\right]$ in nanoconfinement, QENS spectra over the full available energy transfer range were acquired at temperatures, selected on the basis of the fixed window scans (see exemplary spectra from both spectrometers in Fig. 8). At IN16B spectra up to $350 \mathrm{~K}$ were measured, while at FOCUS with its lower resolution but wider energy window, a temperature range from $310 \mathrm{~K}$ to $390 \mathrm{~K}$ is covered. For the analysis of the resulting QENS spectra the following dynamic structure factor is applied:

$$
S(Q, \Delta E)=f \cdot \delta(\Delta E)+(1-f) \cdot S^{\mathrm{dyn}}(Q, \Delta E),
$$

with

$$
S^{\mathrm{dyn}}(Q, \Delta E)=S_{1}(Q, \Delta E) \otimes S_{2}(Q, \Delta E) .
$$

To $S(Q, \Delta E)$ a linear background is added and for the fit to the data, it is further convoluted with the resolution function of the instrument, using a measurement of the particular sample at $2 \mathrm{~K}$ or $40 \mathrm{~K}$ in the case of IN16B and FOCUS, respectively. In the spectra a considerable part of elastically scattered

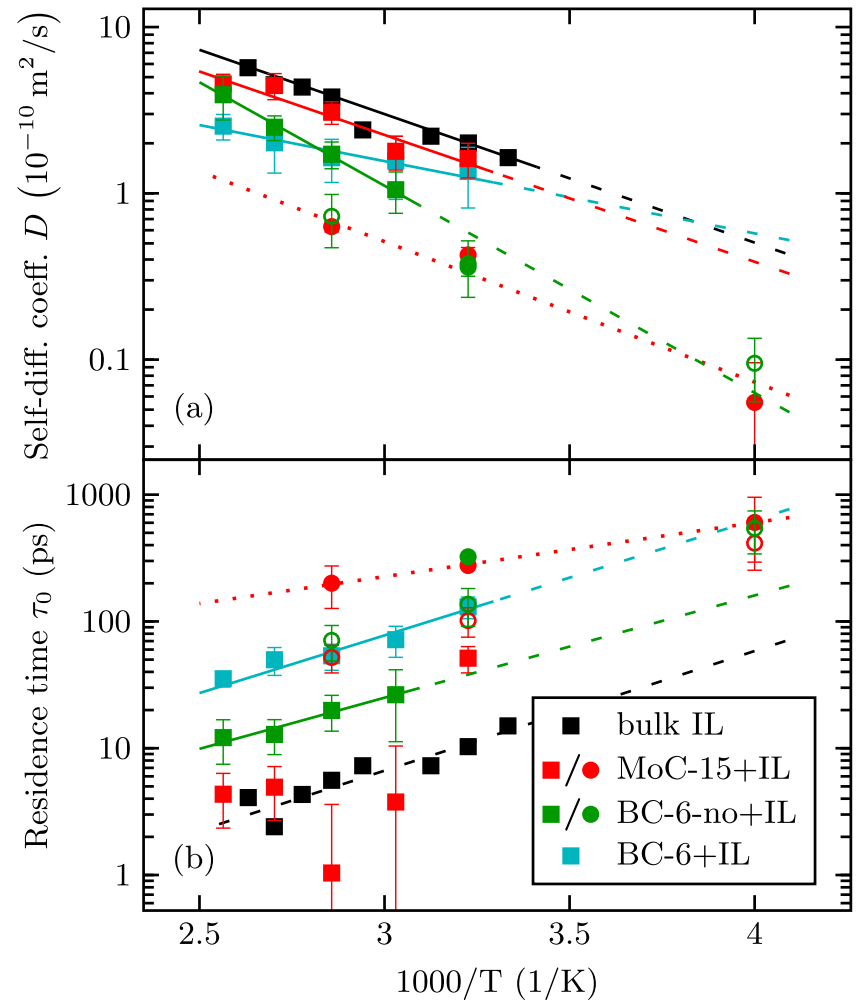

FIG. 7. (a) Self-diffusion coefficients $D$ and (b) residence times $\tau_{0}$ of the $[\mathrm{BuPy}]$ cation, as a function of temperature for different nanopore sizes, determined using the Singwi-Sjölander jumpdiffusion model. Two translational diffusive processes on different time scales are observed. Squares denote data from the FOCUS timeof-flight spectrometer and circles such from the IN16B backscattering spectrometer, at which open circles show values derived from the inelastic fixed window scans. Solid lines are fits with an Arrhenius temperature dependence [see, e.g., Eq. (8)]. Dashed/dotted lines are guides for the eye. Bulk data are taken from Embs et al. [37].

neutrons is observed. Therefore, the fraction $f$ of immobile scatterers is needed to be incorporated within the scattering law. To keep the model computationally as stable as possible, the elastic fraction is considered for both components of the scattering law, i.e., these cations are assumed to exhibit neither translational diffusion nor localized motions. The scattering laws $S_{1}$ and $S_{2}$ are fully analog to $S^{\text {local }}$ and $S^{\text {global }}$ used for the analysis of the fixed window scans [see Eqs. (1) and (6)]. However, the HWHM $\Gamma_{1}$ and $\Gamma_{2}$ of the corresponding Lorentzians are treated as free parameters now.

The line width $\Gamma_{1}$ of the data acquired at FOCUS is found to be in the range of $60-250 \mu \mathrm{eV}$, depending on the temperature, and shows no clear wave vector dependence. It has a comparable magnitude as in the case of the bulk liquid $[37,40]$ with a tendency to lower values in the confinement. Furthermore, it exhibits a certain pore size dependence, hinting towards slower dynamics in narrower pores. This component in these QENS spectra is ascribed to localized motions, like the rotation of methyl end groups [37]. $\Gamma_{2}$ the HWHM of the second component, however, is found to have a $Q$ dependence according to the Singwi-Sjölander jump-diffusion model [see Eq. (7)]. The corresponding selfdiffusion coefficients $D$ and residence times $\tau_{0}$, determined 


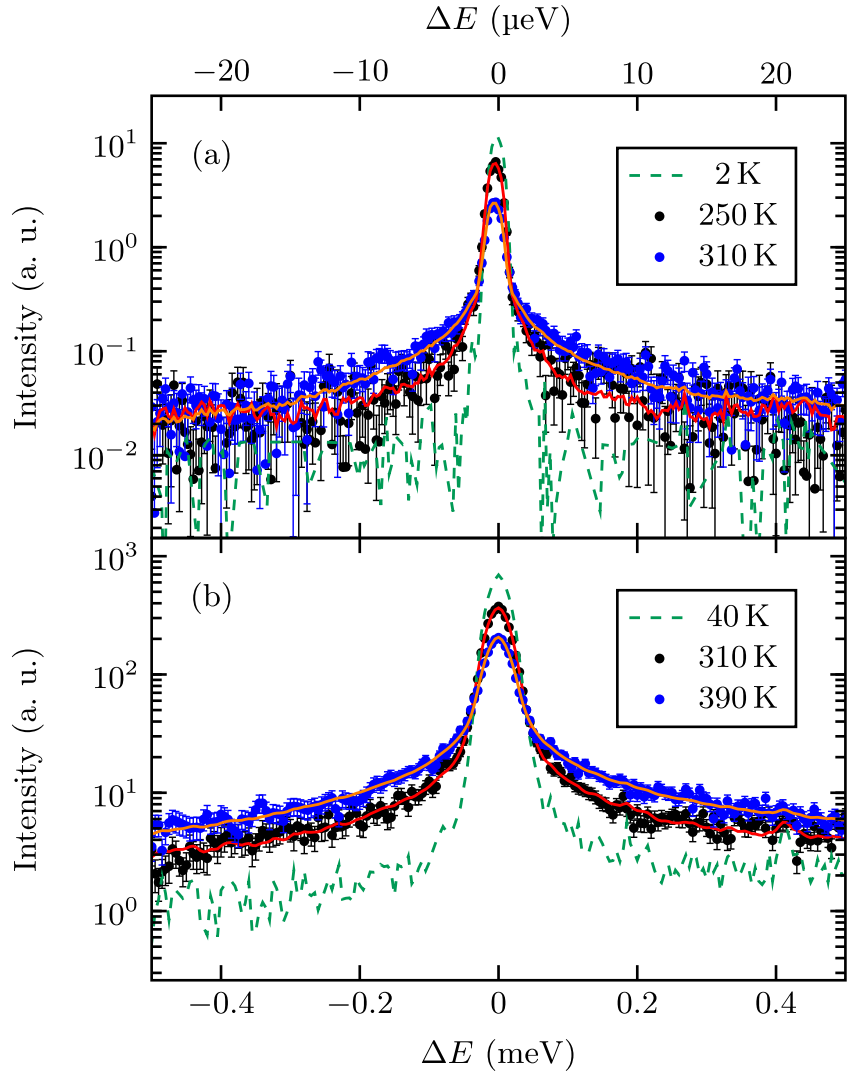

FIG. 8. Selected QENS spectra for the ionic-liquid-filled nanoporous carbon MoC-15 as obtained from (a) IN16B ( $Q=$ $\left.1.18 \AA^{-1}\right)$ and (b) FOCUS $\left(Q=1.26 \AA^{-1}\right)$. Solid lines denote the best fit to the data with the model as described in the text.

by a fit of the model to the data, are shown as squares in Fig. 7 as a function of temperature for the samples of different pore sizes. For sample SiC-2 unfortunately no reliable information about the self-diffusion dynamics of the ionic liquid could be derived from the QENS spectra.

As one can see, the self-diffusion coefficients are notably smaller compared to the bulk values and show a pore size dependence in a way, that with decreasing size of the confinement also $D$ decreases. Alike, the residence time increases with decreasing pore size. Both the self-diffusion coefficient and the residence time exhibit an Arrhenius-like temperature dependence but with different activation energies.

This is in contrast to the assumptions for the analysis of the IFWS and might explain the differing activation energies obtained from the full spectra, where in deviation from the IFWS results no clear pore-size dependence is found. For the activation energy related to the self-diffusion coefficient of $[\mathrm{BuPy}]\left[\mathrm{Tf}_{2} \mathrm{~N}\right]$ within the nanoconfinement of MoC-15 a value of $15 \mathrm{~kJ} / \mathrm{mol}$ is found. For BC-6-no and BC-6 activation energies of $23 \mathrm{~kJ} / \mathrm{mol}$ and $9 \mathrm{~kJ} / \mathrm{mol}$ are determined, respectively. At first, this appears to be surprising, since according to Fig. 1, both samples have a very similar distribution of pore widths. However, they underwent different synthesis pathways: BC-6 was air oxidized before the final vacuum annealing step, while BC-6-no was not. The introduction of surface oxide in this intermediate step likely changes the carbon-carbon bonding, such that it is reasonable to assume that this resulted in different pore morphologies and pore walls roughnesses during the vacuum annealing, when surface functional groups-like oxygen-are removed. Indeed, there are hints from molecular dynamics simulations that such differences influence the mesoscopic structure and dynamics of an ionic liquid in carbon nanoconfinement [65-67]. Also the broad pore-size distribution is presumably of relevance here, because the QENS methods measure the dynamics averaged over all pores. Consequently, also the associated activation energies underlie such an averaging. While the translational diffusion of the ionic liquid in the pores of MoC15 has an activation energy similar to the bulk value of 12.3 $14.8 \mathrm{~kJ} / \mathrm{mol}[37,38,40,41]$, the one for BC-6-no is higher, whereas it is smaller for BC-6. It is imaginable that the interaction with the pore wall and structural changes of the ionic liquid that are provoked by the respective pore morphology lead to an alteration of the ionic liquid's dynamics and the related activation energies. Indeed, an activation energy reduction of the translational self-diffusion with respect to the bulk is for example found for hexane in porous silica, where it further decreases with decreasing pore size [68]. Alike for supercooled water in silica nanopores such a pore-size dependence of the activation energy is observed [69].

Similarly in the case of the data acquired on IN16B with its higher resolution but smaller dynamic range compared to FOCUS, two dynamic components are found. One of these is ascribed to the superposition of the slow localized dynamics, as found from the IFWS, and the diffusive motion, as seen with FOCUS. However, since they possess both a comparable HWHM, a separation of both components is challenging and the analysis is focused to the second, much narrower component, here. The latter is of diffusive nature and its HWHM exhibits again a behavior, following the Singwi-Sjölander jump-diffusion model. The corresponding self-diffusion coefficients obtained from these IN16B spectra are almost one order of magnitude smaller (see filled circles in Fig. 7(a)] than those found with FOCUS, when observing molecular motions at a different time scale. The respective residence time $\tau_{0}$ on the other hand is increased by one order of magnitude. The self-diffusion coefficients determined from full QENS spectra (more sensitive to spectral shape) are in a very good agreement with those derived from the IFWS (more sensitive to temperature behavior) above [see open circles in Fig. 7(a)]. However, it should be noted that the dynamics inferred from the IFWS are the result of a simplified model containing only one component for each, the center-of-mass diffusion of the whole cation and the localized dynamics, respectively. Consequently, the derived dynamic properties need to be considered as averaged over the fast and the slow species.

Interestingly, we see not only two translational diffusive motions at different time scales, but also a fast and a slow localized dynamic, ascribed amongst others to methyl group motions. It is reasonable to assume that cations exhibiting a sluggish translational diffusion are also those that correspondingly possess slow localized dynamics. The existence of several translational diffusive motions on different dynamic length and time scales is not unusual for ionic liquids in carbon nanoconfinement $[13,14]$ and even in the bulk state 


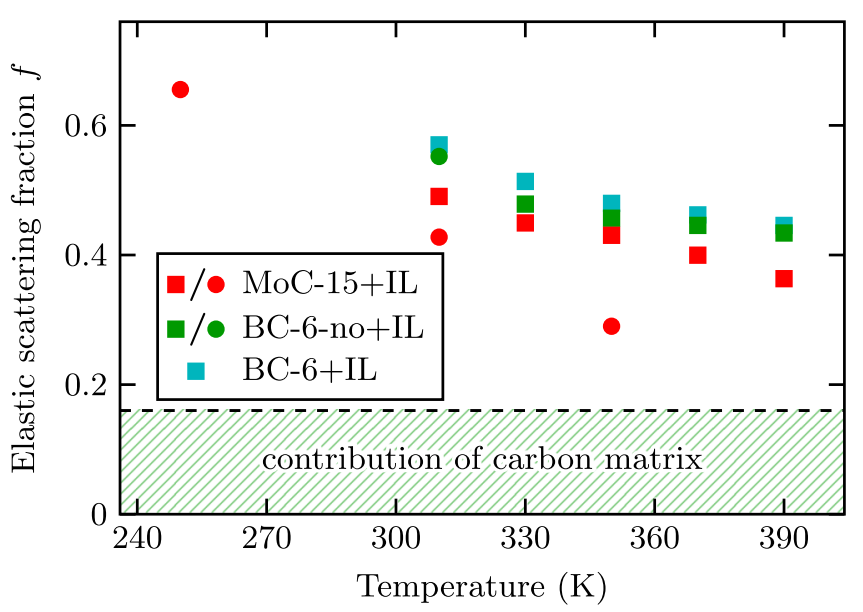

FIG. 9. Fraction $f$ of elastic scattering in the quasielastic spectra, resulting from immobilized cations of the ionic liquid. Squares denote the fast diffusive process as seen with FOCUS and circles the slower one, observed with IN16B.

[70-72], where it is often related to a nanoscale structural organization [73-75]. Also for bulk $[\mathrm{BuPy}]\left[\mathrm{Tf}_{2} \mathrm{~N}\right]$ such a dynamic heterogeneity is found [40]. In these cases, one dynamic component is regarded as the diffusion of ions bound in ionic aggregates, while the other, long range diffusive motion is considered to take place in the free liquid between these clusters $[40,70,70-72]$. In this connection, a disturbance of the Coulombic charge ordering inside conductive pores, like those of carbon materials, is found, e.g., by x-ray diffraction experiments and Monte Carlo simulations [76,77], at which this effect is stronger in smaller pores [26,76,78]. Molecular dynamics simulations deliver a further interpretation approach $[18,79]$. They find that ions in molecular layers being closer to the pore walls, compared to those in the pore center, exhibit decreased diffusion coefficients and thus give rise to heterogeneous cation dynamics $[18,79]$.

At all temperatures the measured quasielastic spectra at both spectrometers contain a considerable fraction of elastically scattered neutrons, as already mentioned above. While only a small part of this can be ascribed to the carbon matrix (see estimation [80] of this contribution in Figs. 4 and 9), the predominant portion originates from nonmoving cations of $[\mathrm{BuPy}]\left[\mathrm{Tf}_{2} \mathrm{~N}\right]$. This immobile fraction is decreasing with rising temperature, but even at $390 \mathrm{~K}$, i.e. around $90 \mathrm{~K}$ above the bulk melting point, there is still a notable fraction of immobilized cations. The formation of pore-wall-adsorbed, immobile surface layers is a well-known phenomenon of complex molecules in nanoconfinement [51,63,81-83], as is in the case of ionic liquids in nanoporous carbons $[14,14,20,23,24]$. From Fig. 9 it appears that there are only a few differences between the different specimens concerning the immobile fraction. One of the reasons could be the large volume fraction of micropores, present in all samples, where the cations might be expected to remain largely immobile. However, there seems to be a weak tendency towards a higher portion in samples with smaller pores, although one should be aware of the uncertainties due to the carbon matrix contribution [80]. Nevertheless, such a trend is reasonable, because in narrower pores the surface-to-volume ratio is higher and therefore these immobile surface layers occupy a larger fraction of the overall pore volume. The pore-size dependence of the immobile fraction is also supported by the EFWS in Fig. 4(a). While the elastic intensity of MoC-15+IL with its comparatively large pores appears to saturate at high temperatures only somewhat above the elastic contribution of the carbon matrix, the saturation value of the elastic intensity for the samples with smaller pores is considerably higher and increasing with decreasing pore size. The immobile fraction concerning those molecules exhibiting the slower of the two translational diffusive motions in the nanoconfined ionic liquid appears to be lower than that of the faster diffusing cations (cp. circles and squares in Fig. 9). This is because the time scale of the slow dynamics is below the resolution of FOCUS and thus they appear to be immobile there, giving rise to a seemingly elastic contribution to the scattering signal, while they are seen as mobile within the higher resolution of IN16B.

\section{CONCLUSIONS}

The cation dynamics of a room-temperature ionic liquid under the nanoconfinement of porous carbons with different pore sizes have been analyzed as a function of temperature using quasielastic neutron scattering techniques. It is shown in a pioneering manner that the analysis of the fixed window scans gives already a quite comprehensive overview over the different dynamic processes appearing at the nano- and picosecond time scale and about their alteration in confinement, when compared to much more time-consuming full QENS spectroscopic data. A further investigation of the full spectroscopic information finds two diffusive motions of the cation on different dynamic time scales, both slower than in the bulk liquid, while a considerable fraction of molecules stays immobile in the restricting carbon nanopores over the whole temperature range. The obtained self-diffusion coefficients are found to exhibit an Arrhenius-like temperature dependence. But not only the translational diffusion of the whole molecule is influenced by the nanoconfinement. Also the localized dynamics of parts of the cation are successively slowed down with decreasing pore size, while the activation energy of this dynamic process also decreases.

Interestingly, our experiments on the thermally excited stochastic motions of the cations unambiguously indicate that the ionic liquid in the nanoporous carbon melts already well below the bulk melting point. Thus, we find no hints of confinement-induced freezing of ionic liquids as recently inferred from slit-pore confinement [25]. By contrast we observe the opposite, clear premelting similarly as is known for many conventional liquids $[51,54,55]$ and this extends the operating temperature range for potential technical applications of such systems, like supercapacitors.

From a more materials design perspective our study provides mechanistic insights on the slow dynamics of ionic liquids in micropores. It motivates confinement for lowtemperature applications, however, it also suggests that hierarchical pore structures, where micropores coexist with mesopores to simultaneously optimize self-diffusive transport and thus functional dynamics with high specific inner surface areas and thus electrical capacities [84]. In particular, 
hierarchical pore structures with pore size distributions mimicking bio-inspired Murray materials, hierarchical materials where the pore size distribution is chosen to optimize this tradeoff [85], similarly as established in biological vascularizations, could be able to solve this dichotomy.

Finally, we envision some further analysis on the nature of the two diffusive dynamics of the cation. The structural and dynamic investigation of the ionic liquid inside directed nanopores with well defined pore shapes and sizes appear promising. In particular, our findings of distinct thermal activation energies for molecular self-diffusion in nanoporous carbons with similar pore size indicate the importance of the pore morphology and roughness on the molecular mobility, beyond the pure confinement size. This observation motivates a systematic study of these geometrical parameters on the mobility in combination with molecular dynamics simulations [86].

In addition the influence of an applied electrical potential on the molecular mobility of ionic liquids in carbon nanoconfinement needs more attention [87]. Furthermore, mixtures of ionic liquids with solvents may lead to an optimized diffusivity and ionic conductivity $[88,89]$ and should be systematically investigated concerning their dynamics in carbon nanopores. Also the exploration of the self-diffusion dynamics of aqueous electrolytes confined in nanoporous carbon materials by neutron spectroscopy could be particularly interesting with respect to the impact of ion confinement and desolvation of ions in confinement in the future [90,91].

\section{ACKNOWLEDGMENTS}

We are indebted to Alexei Kornyshev (Imperial College, London) and Yury Gogotsi (Drexel University, Philadelphia) for useful discussions. Furthermore, we thank the working group of Rolf Hempelmann (Saarland University) for providing the ionic liquid. M.B. and P.H. acknowledge support by the German Research Foundation (Deutsche Forschungsgemeinschaft, DFG) within the project "Property Changes of Multiphasic Fluids by Geometrical Confinement in Advanced Mesoporous Materials" (Project No. 407319385) and the DFG Graduate School GRK 2462 "Processes in natural and technical Particle-Fluid-Systems (PintPFS)" (Project No. 390794421).
[1] P. Simon and Y. Gogotsi, Materials for electrochemical capacitors, Nat. Mater. 7, 845 (2008).

[2] Y. Zhai, Y. Dou, D. Zhao, P. F. Fulvio, R. T. Mayes, and S. Dai, Carbon materials for chemical capacitive energy storage, Adv. Mater. 23, 4828 (2011).

[3] S. Zhang, J. Zhang, Y. Zhang, and Y. Deng, Nanoconfined ionic liquids, Chem. Rev. 117, 6755 (2017).

[4] N. C. Osti and E. Mamontov, Microscopic dynamics in roomtemperature ionic liquids confined in materials for supercapacitor applications, Sustainable Energy \& Fuels 4, 1554 (2020).

[5] P. Huber, ed., Soft Matter under Geometrical Confinement: From Fundamentals at Planar Surfaces and Interfaces to Functionalities of Nanoporous Materials, Soft Matter and Biomaterials on the Nanoscale, Vol. 1 (World Scientific, Singapore, 2020).

[6] M. Armand, F. Endres, D. R. MacFarlane, H. Ohno, and B. Scrosati, Ionic-liquid materials for the electrochemical challenges of the future, Nat. Mater. 8, 621 (2009).

[7] J. Vatamanu, O. Borodin, M. Olguin, G. Yushin, and D. Bedrov, Charge storage at the nanoscale: understanding the trends from the molecular scale perspective, J. Mater. Chem. A 5, 21049 (2017).

[8] M. Salanne, Ionic liquids for supercapacitor applications, Top. Curr. Chem. 375, 63 (2017).

[9] J. Cherusseri, K. S. Kumar, N. Choudhary, N. Nagaiah, Y. Jung, T. Roy, and J. Thomas, Novel mesoporous electrode materials for symmetric, asymmetric and hybrid supercapacitors, Nanotechnology 30, 202001 (2019).

[10] S. Zhang, N. Sun, X. He, X. Lu, and X. Zhang, Physical properties of ionic liquids: Database and evaluation, J. Phys. Chem. Ref. Data 35, 1475 (2006).

[11] A. Nazet, S. Sokolov, T. Sonnleitner, S. Friesen, and R. Buchner, Densities, refractive indices, viscosities, and conductivities of non-imidazolium ionic liquids
[Et $\left.{ }_{3} \mathrm{~S}\right][\mathrm{TFSI}],\left[\mathrm{Et}_{2} \mathrm{MeS}\right][\mathrm{TFSI}],[\mathrm{BuPy}][\mathrm{TFSI}],\left[\mathrm{N}_{8881}\right][\mathrm{TFA}]$, and $\left[\mathrm{P}_{14}\right][\mathrm{DCA}]$, J. Chem. Eng. Data 62, 2549 (2017).

[12] K. S. Panesar, C. Hugon, G. Aubert, P. Judeinstein, J.-M. Zanotti, and D. Sakellariou, Measurement of self-diffusion in thin samples using a novel one-sided NMR magnet, Microporous Mesoporous Mater. 178, 79 (2013).

[13] S. M. Chathoth, E. Mamontov, S. Dai, X. Wang, P. F. Fulvio, and D. J. Wesolowski, Fast diffusion in a room temperature ionic liquid confined in mesoporous carbon, Europhys. Lett. 97, 66004 (2012)

[14] S. M. Chathoth, E. Mamontov, P. F. Fulvio, X. Wang, G. A. Baker, S. Dai, and D. J. Wesolowski, An unusual slowdown of fast diffusion in a room temperature ionic liquid confined in mesoporous carbon, Europhys. Lett. 102, 16004 (2013).

[15] V. V. Chaban and O. V. Prezhdo, Nanoscale carbon greatly enhances mobility of a highly viscous ionic liquid, ACS Nano 8, 8190 (2014).

[16] Q. Berrod, F. Ferdeghini, P. Judeinstein, N. Genevaz, R. Ramos, A. Fournier, J. Dijon, J. Ollivier, S. Rols, D. Yu et al., Enhanced ionic liquid mobility induced by confinement in 1D CNT membranes, Nanoscale 8, 7845 (2016).

[17] R. Singh, J. Monk, and F. R. Hung, A computational study of the behavior of the ionic liquid $\left[\mathrm{BMIM}^{+}\right]\left[\mathrm{PF}_{6}^{-}\right]$confined inside multiwalled carbon nanotubes, J. Phys. Chem. C 114, 15478 (2010).

[18] S. Li, K. S. Han, G. Feng, E. W. Hagaman, L. Vlcek, and P. T. Cummings, Dynamic and structural properties of roomtemperature ionic liquids near silica and carbon surfaces, Langmuir 29, 9744 (2013).

[19] B. Dyatkin, Y. Zhang, E. Mamontov, A. I. Kolesnikov, Y. Cheng, H. M. Meyer, P. T. Cummings, and Y. Gogotsi, Influence of surface oxidation on ion dynamics and capacitance in porous and nonporous carbon electrodes, J. Phys. Chem. C 120, 8730 (2016). 
[20] B. Dyatkin, N. C. Osti, Y. Zhang, H.-W. Wang, E. Mamontov, W. T. Heller, P. Zhang, G. Rother, P. T. Cummings, D. J. Wesolowski, and Y. Gogotsi, Ionic liquid structure, dynamics, and electrosorption in carbon electrodes with bimodal pores and heterogeneous surfaces, Carbon 129, 104 (2018).

[21] M. Mezger, H. Schröder, H. Reichert, S. Schramm, J. S. Okasinski, S. Schöder, V. Honkimäki, M. Deutsch, B. M. Ocko, J. Ralston, M. Rohwerder, M. Stratmann, and H. Dosch, Molecular layering of fluorinated ionic liquids at a charged sapphire (0001) surface, Science 322, 424 (2008).

[22] L. R. Griffin, K. L. Browning, S. M. Clarke, A. M. Smith, S. Perkin, M. W. A. Skoda, and S. E. Norman, Direct measurements of ionic liquid layering at a single mica-liquid interface and in nano-films between two mica-liquid interfaces, Phys. Chem. Chem. Phys. 19, 297 (2017).

[23] J. L. Bañuelos, G. Feng, P. F. Fulvio, S. Li, G. Rother, N. Arend, A. Faraone, S. Dai, P. T. Cummings, and D. J. Wesolowski, The influence of a hierarchical porous carbon network on the coherent dynamics of a nanoconfined room temperature ionic liquid: A neutron spin echo and atomistic simulation investigation, Carbon 78, 415 (2014).

[24] B. Dyatkin, N. C. Osti, A. Gallegos, Y. Zhang, E. Mamontov, P. T. Cummings, J. Wu, and Y. Gogotsi, Electrolyte cation length influences electrosorption and dynamics in porous carbon supercapacitors, Electrochim. Acta 283, 882 (2018).

[25] J. Comtet, A. Niguès, V. Kaiser, B. Coasne, L. Bocquet, and A. Siria, Nanoscale capillary freezing of ionic liquids confined between metallic interfaces and the role of electronic screening, Nat. Mater. 16, 634 (2017).

[26] S. Kondrat, P. Wu, R. Qiao, and A. A. Kornyshev, Accelerating charging dynamics in subnanometre pores, Nat. Mater. 13, 387 (2014).

[27] G. Feng and P. T. Cummings, Supercapacitor capacitance exhibits oscillatory behavior as a function of nanopore size, J. Phys. Chem. Lett. 2, 2859 (2011).

[28] D.-e. Jiang, Z. Jin, and J. Wu, Oscillation of capacitance inside nanopores, Nano Lett. 11, 5373 (2011).

[29] P. Wu, J. Huang, V. Meunier, B. G. Sumpter, and R. Qiao, Complex capacitance scaling in ionic liquids-filled nanopores, ACS Nano 5, 9044 (2011).

[30] H. Tokuda, S. Tsuzuki, M. A. B. H. Susan, K. Hayamizu, and M. Watanabe, How ionic are room-temperature ionic liquids? An indicator of the physicochemical properties, J. Phys. Chem. B 110, 19593 (2006).

[31] M. Kanakubo, Y. Hiejima, K. Minami, T. Aizawa, and H. Nanjo, Melting point depression of ionic liquids confined in nanospaces, Chem. Commun. 42, 1828 (2006).

[32] D. Weingarth, R. Drumm, A. Foelske-Schmitz, R. Kötz, and V. Presser, An electrochemical in situ study of freezing and thawing of ionic liquids in carbon nanopores, Phys. Chem. Chem. Phys. 16, 21219 (2014).

[33] V. Presser, M. Heon, and Y. Gogotsi, Carbide-derived carbons from porous networks to nanotubes and graphene, Adv. Funct. Mater. 21, 810 (2011).

[34] B. Dyatkin and Y. Gogotsi, Effects of structural disorder and surface chemistry on electric conductivity and capacitance of porous carbon electrodes, Faraday Discuss. 172, 139 (2014).

[35] B. Dyatkin, E. Mamontov, K. M. Cook, and Y. Gogotsi, Capacitance, charge dynamics, and electrolyte-surface interactions in functionalized carbide-derived carbon electrodes,
Progress Natural Science: Materials International 25, 631 (2015).

[36] A. V. Neimark, Y. Lin, P. I. Ravikovitch, and M. Thommes, Quenched solid density functional theory and pore size analysis of micro-mesoporous carbons, Carbon 47, 1617 (2009).

[37] J. P. Embs, T. Burankova, E. Reichert, and R. Hempelmann, Cation dynamics in the pyridinium based ionic liquid 1$n$-butylpyridinium bis((trifluoromethyl)sulfonyl) as seen by quasielastic neutron scattering, J. Phys. Chem. B 116, 13265 (2012).

[38] J. P. Embs, T. Burankova, E. Reichert, V. Fossog, and R. Hempelmann, QENS study of diffusive and localized cation motions of pyridinium-based ionic liquids, J. Phys. Soc. Jpn. 82, SA003 (2013).

[39] T. Burankova, E. Reichert, V. Fossog, R. Hempelmann, and J. P. Embs, The dynamics of cations in pyridinium-based ionic liquids by means of quasielastic- and inelastic neutron scattering, J. Mol. Liq. 192, 199 (2014).

[40] T. Burankova, R. Hempelmann, A. Wildes, and J. P. Embs, Collective ion diffusion and localized single particle dynamics in pyridinium-based ionic liquids, J. Phys. Chem. B 118, 14452 (2014).

[41] T. Burankova, G. Simeoni, R. Hempelmann, J. F. Mora Cardozo, and J. P. Embs, Dynamic heterogeneity and flexibility of the alkyl chain in pyridinium-based ionic liquids, J. Phys. Chem. B 121, 240 (2017).

[42] M. Busch, J. P. Embs, B. Frick, T. Hofmann, and P. Huber, Dynamics of an ionic liquid confined in nanoporous carbon substrates, Institut Laue-Langevin (ILL): Grenoble, 2015; DOI:10. 5291/ILL-DATA.6-02-558.

[43] B. Frick, J. Combet, and L. van Eijck, New possibilities with inelastic fixed window scans and linear motor Doppler drives on high resolution neutron backscattering spectrometers, Nucl. Instrum. Methods Phys. Res., Sect. A 669, 7 (2012).

[44] V. F. Sears, Neutron scattering lengths and cross sections, Neutron News 3, 26 (1992).

[45] R. T. Azuah, L. R. Kneller, Y. Qiu, P. L. W. Tregenna-Piggott, C. M. Brown, J. R. D. Copley, and R. M. Dimeo, DAVE: A comprehensive software suite for the reduction, visualization, and analysis of low energy neutron spectroscopic data, J. Res. Natl. Inst. Stand. Technol. 114, 341 (2009).

[46] D. Richard, M. Ferrand, and G. J. Kearley, Analysis and visualisation of neutron-scattering data, J. Neutron Res. 4, 33 (1996).

[47] A. Noda, K. Hayamizu, and M. Watanabe, Pulsed-gradient spinecho ${ }^{1} \mathrm{H}$ and ${ }^{19} \mathrm{~F}$ NMR ionic diffusion coefficient, viscosity, and ionic conductivity of non-chloroaluminate room-temperature ionic liquids, J. Phys. Chem. B 105, 4603 (2001).

[48] H. Tokuda, K. Ishii, M. A. B. H. Susan, S. Tsuzuki, K. Hayamizu, and M. Watanabe, Physicochemical properties and structures of room-temperature ionic liquids. 3. variation of cationic structures, J. Phys. Chem. B 110, 2833 (2006).

[49] K. Knorr, P. Huber, and D. Wallacher, Thermodynamic and structural investigations of condensates of small molecules in mesopores, Z. Phys. Chem. 222, 257 (2008).

[50] C. Schaefer, T. Hofmann, D. Wallacher, P. Huber, and K. Knorr, Melting and Freezing of Argon in a Granular Packing of Linear Mesopore Arrays, Phys. Rev. Lett. 100, 175701 (2008).

[51] P. Huber, Soft matter in hard confinement: phase transition thermodynamics, structure, texture, diffusion and flow 
in nanoporous media, J. Phys.: Condens. Matter 27, 103102 (2015).

[52] M. Maruyama, M. Bienfait, J. G. Dash, and G. Coddens, Interfacial melting of ice in graphite and talc powders, J. Cryst. Growth 118, 33 (1992).

[53] P. Huber and K. Knorr, Adsorption-desorption isotherms and $\mathrm{x}$-ray diffraction of Ar condensed into a porous glass matrix, Phys. Rev. B 60, 12657 (1999).

[54] H. K. Christenson, Confinement effects on freezing and melting, J. Phys.: Condens. Matter 13, R95 (2001).

[55] C. Alba-Simionesco, B. Coasne, G. Dosseh, G. Dudziak, K. E. Gubbins, R. Radhakrishnan, and M. Sliwinska-Bartkowiak, Effects of confinement on freezing and melting, J. Phys.: Condens. Matter 18, R15 (2006).

[56] C. L. Jackson and G. B. McKenna, The melting behavior of organic materials confined in porous solids, J. Chem. Phys. 93, 9002 (1990).

[57] M. Bée, Quasielastic Neutron Scattering - Principles and Applications in Solid State Chemistry, Biology and Materials Science, edited by D. J. Millen (Adam Hilger, Bristol and Philadelphia, 1988).

[58] H. H. Grapengeter, B. Alefeld, and R. Kosfeld, An investigation of micro-brownian motions in polydimethylsiloxane by complementary incoherent-neutron-scattering and nuclearmagnetic-resonance experiments below room temperature, Colloid Polym. Sci. 265, 226 (1987).

[59] A. Chahid, A. Alegria, and J. Colmenero, Methyl group dynamics in poly(vinyl methyl ether). A rotation rate distribution model, Macromolecules 27, 3282 (1994).

[60] R. E. Lechner, H. J. Bleif, H. Dachs, R.. Marx, M. Stahn, and I. Anderson, Two-dimensional proton diffusion in $\mathrm{CsH}_{3} \mathrm{O}_{2}$, Solid State Ionics 46, 25 (1991).

[61] K. S. Singwi and A. Sjölander, Diffusive motions in water and cold neutron scattering, Phys. Rev. 119, 863 (1960).

[62] A. J. Moreno, J. Colmenero, A. Alegría, C. Alba-Simionesco, G. Dosseh, D. Morineau, and B. Frick, Methyl group dynamics in a confined glass, Eur. Phys. J. E 12, 43 (2003).

[63] B. Frick, C. Alba-Simionesco, G. Dosseh, C. Le Quellec, A. J. Moreno, J. Colmenero, A. Schönhals, R. Zorn, K. Chrissopoulou, S. H. Anastasiadis, and K. Dalnoki-Veress, Inelastic neutron scattering for investigating the dynamics of confined glass-forming liquids, J. Non-Cryst. Solids 351, 2657 (2005), proceedings of 3rd International Conference on Broadband Dielectric Spectroscopy and its Applications; Section 3. Dynamics of nano-confined systems.

[64] A. Schönhals, H. Goering, C. Schick, B. Frick, and R. Zorn, Polymers in nanoconfinement: What can be learned from relaxation and scattering experiments?, J. Non-Cryst. Solids 351, 2668 (2005), proceedings of 3rd International Conference on Broadband Dielectric Spectroscopy and its Applications.

[65] J. Monk, R. Singh, and F. R. Hung, Effects of pore size and pore loading on the properties of ionic liquids confined inside nanoporous CMK-3 carbon materials, J. Phys. Chem. C 115, 3034 (2011).

[66] J. Vatamanu, L. Cao, O. Borodin, D. Bedrov, and G. D. Smith, On the influence of surface topography on the electric double layer structure and differential capacitance of graphite/ionic liquid interfaces, J. Phys. Chem. Lett. 2, 2267 (2011).
[67] J. Seebeck, P. Schiffels, S. Schweizer, J.-R. Hill, and R. H. Meißner, Electrical double layer capacitance of curved graphite electrodes, J. Phys. Chem. C 124, 5515 (2020).

[68] J. Baumert, B. Asmussen, C. Gutt, and R. Kahn, Pore-size dependence of the self-diffusion of hexane in silica gels, J. Chem. Phys. 116, 10869 (2002).

[69] L. Liu, S.-H. Chen, A. Faraone, C.-W. Yen, C.-Y. Mou, A. I. Kolesnikov, E. Mamontov, and J. Leao, Quasielastic and inelastic neutron scattering investigation of fragileto-strong crossover in deeply supercooled water confined in nanoporous silica matrices, J. Phys.: Condens. Matter 18, S2261 (2006).

[70] F. Ferdeghini, Q. Berrod, J.-M. Zanotti, P. Judeinstein, V. G. Sakai, O. Czakkel, P. Fouquet, and D. Constantin, Nanostructuration of ionic liquids: impact on the cation mobility. a multiscale study, Nanoscale 9, 1901 (2017).

[71] Q. Berrod, F. Ferdeghini, J.-M. Zanotti, P. Judeinstein, D. Lairez, V. García Sakai, O. Czakkel, P. Fouquet, and D. Constantin, Ionic liquids: evidence of the viscosity scaledependence, Sci. Rep. 7, 2241 (2017).

[72] T. Burankova, J. F. Mora Cardozo, D. Rauber, A. Wildes, and J. P. Embs, Linking structure to dynamics in protic ionic liquids: A neutron scattering study of correlated and single-particle motions, Sci. Rep. 8, 16400 (2018).

[73] A. Triolo, O. Russina, H.-J. Bleif, and E. Di Cola, Nanoscale segregation in room temperature ionic liquids, J. Phys. Chem. B 111, 4641 (2007).

[74] O. Russina, A. Triolo, L. Gontrani, and R. Caminiti, Mesoscopic structural heterogeneities in room-temperature ionic liquids, J. Phys. Chem. Lett. 3, 27 (2012).

[75] O. Russina and A. Triolo, Ionic liquids and neutron scattering, in Neutron Scattering - Applications in Biology, Chemistry, and Materials Science, Experimental Methods in the Physical Sciences, Vol. 49, edited by F. Fernandez-Alonso and D. L. Price (Academic Press, Cambridge, MA, 2017), Chap. 4, pp. 213-278.

[76] R. Futamura, T. Iiyama, Y. Takasaki, Y. Gogotsi, M. J. Biggs, M. Salanne, J. Ségalini, P. Simon, and K. Kaneko, Partial breaking of the coulombic ordering of ionic liquids confined in carbon nanopores, Nat. Mater. 16, 1225 (2017).

[77] S. Kondrat, N. Georgi, M. V. Fedorov, and A. A. Kornyshev, A superionic state in nano-porous double-layer capacitors: insights from Monte Carlo simulations, Phys. Chem. Chem. Phys. 13, 11359 (2011).

[78] S. Kondrat and A. Kornyshev, Superionic state in doublelayer capacitors with nanoporous electrodes, J. Phys.: Condens. Matter 23, 022201 (2010).

[79] R. Singh, J. Monk, and F. R. Hung, Heterogeneity in the dynamics of the ionic liquid $\left[\mathrm{BMIM}^{+}\right]\left[\mathrm{PF}_{6}^{-}\right]$confined in a slit nanopore, J. Phys. Chem. C 115, 16544 (2011).

[80] Estimated to be in the range of 0.1-0.2. Estimation from the elastic scattering intensity of an empty CDC matrix in comparison to that of an ionic-liquid-filled one with the same amount of $\mathrm{CDC}$, both measured at $2 \mathrm{~K}$, where all neutrons are practically elastically scattered. Comparison of the calculated scattering cross sections of the empty and the ionic-liquid-filled CDC, weighted with the respective particle numbers, confirms this finding. 
[81] A. Kusmin, S. Gruener, A. Henschel, O. Holderer, J. Allgaier, D. Richter, and P. Huber, Evidence of a sticky boundary layer in nanochannels: A neutron spin echo study of $n$-hexatriacontane and poly(ethylene oxide) confined in porous silicon, J. Phys. Chem. Lett. 1, 3116 (2010).

[82] T. Hofmann, D. Wallacher, M. Mayorova, R. Zorn, B. Frick, and P. Huber, Molecular dynamics of n-hexane: A quasi-elastic neutron scattering study on the bulk and spatially nanochannelconfined liquid, J. Chem. Phys. 136, 124505 (2012).

[83] D. Richter and M. Kruteva, Polymer dynamics under confinement, Soft Matter 15, 7316 (2019).

[84] T. Liu, F. Zhang, Y. Song, and Y. Li, Revitalizing carbon supercapacitor electrodes with hierarchical porous structures, J. Mater. Chem. A 5, 17705 (2017).

[85] X. Zheng, G. Shen, C. Wang, Y. Li, D. Dunphy, T. Hasan, C. J. Brinker, and B.-L. Su, Bio-inspired Murray materials for mass transfer and activity, Nat. Commun. 8, 14921 (2017).

[86] P. Scheidler, W. Kob, and K. Binder, Cooperative motion and growing length scales in supercooled confined liquids, Europhys. Lett. 59, 701 (2002).

[87] S. M. Mahurin, E. Mamontov, M. W. Thompson, P. Zhang, C. H. Turner, P. T. Cummings, and S. Dai, Relationship between pore size and reversible and irreversible immobilization of ionic liquid electrolytes in porous carbon under applied electric potential, Appl. Phys. Lett. 109, 14311 (2016).

[88] N. C. Osti, K. L. Van Aken, M. W. Thompson, F. Tiet, D.-e. Jiang, P. T. Cummings, Y. Gogotsi, and E. Mamontov, Solvent polarity governs ion interactions and transport in a solvated room-temperature ionic liquid, J. Phys. Chem. Lett. 8, 167 (2017).

[89] M. W. Thompson, R. Matsumoto, R. L. Sacci, N. C. Sanders, and P. T. Cummings, Scalable screening of soft matter: A case study of mixtures of ionic liquids and organic solvents, J. Phys. Chem. B 123, 1340 (2019).

[90] C. Prehal, C. Koczwara, N. Jäckel, A. Schreiber, M. Burian, H. Amenitsch, M. A. Hartmann, V. Presser, and O. Paris, Quantification of ion confinement and desolvation in nanoporous carbon supercapacitors with modelling and in situ x-ray scattering, Nat. Energy 2, 16215 (2017).

[91] C. Prehal, C. Koczwara, H. Amenitsch, V. Presser, and O. Paris, Salt concentration and charging velocity determine ion charge storage mechanism in nanoporous supercapacitors, Nat. Commun. 9, 4145 (2018). 